Review Article

\title{
Productive and sum graph labelings: a survey*
}

\author{
Lowell W. Beineke ${ }^{1, \dagger}$, Suresh M. Hegde ${ }^{2}$, V. Vilfred Kamalappan ${ }^{3}$ \\ ${ }^{1}$ Department of Mathematical Sciences, Purdue University Fort Wayne, Fort Wayne, Indiana, USA \\ ${ }^{2}$ Department of Mathematical and Computational Sciences, National Institute of Technology Karnataka, Surathkal, Mangalore, India \\ ${ }^{3}$ Department of Mathematics, Central University of Kerala, Kasaragod, Kerala, India
}

(Received: 30 June 2020. Accepted: 24 July 2020. Published online: 11 March 2021.)

(c) 2021 the authors. This is an open access article under the CC BY (International 4.0) license (www.creativecommons.org/licenses/by/4.0/).

\begin{abstract}
Graph labeling is one of the most popular and dynamic areas of graph theory, perhaps even among all of mathematics. The standard problem involves a graph having labels from a given set of integers on its vertices and then are assigned values according to some formula. Frank Harary introduced an alternative problem, in which the labels on the vertices themselves must meet a specified condition. Here, we give a survey of a problem of each type: (a) strongly multiplicative graphs, where, given a labeling of the vertices, each edge is labeled with the product of its vertex labels; (b) sum graphs, where, given a labeling of the vertices, two are adjacent if the sum of their labels is also a vertex label.
\end{abstract}

Keywords: graph labelings; strongly multiplicative graphs; productive graphs; strongly quotient graphs; sum graphs; integral sum graphs.

2020 Mathematics Subject Classification: 05C78, 05C75, 05C07, $05 \mathrm{C} 12$.

\section{Introduction}

Most graph labeling problems have three ingredients: for a given graph $G$ :

- A set $S$ of numbers from which vertex labels are chosen.

- A rule that assigns a value to each edge.

- A condition that these values must satisfy.

Following standard notation, a graph $G$ will generally consist here of a set $V$ of $n$ vertices and a set $E$ of $m$ edges, and will be simple (that is no loops or multiple edges). In a labeling of a graph, the label on a vertex $v$ is denoted $\ell(v)$ and the value on an edge $e$ is denoted $f(e)$. Throughout this article, it is always a requirement that no two vertices in a labeling of a graph receive the same label. Two classical labelings, called "graceful" and "harmonious" labelings, originated in the 1960s. After considering them briefly, we will move on to two that are less well-known. In the spirit of the aforementioned names and their catchiness and in honor of Frank Harary and his mathematical productivity, we call the first of these "productive" here, instead of their original name of "strongly multiplicative". The second type that we consider here are "sum" graphs, given that name by Harary himself.

Information on the subject of graph labeling can be found at the wonderful site founded and maintained by Joe Gallian, "A Dynamic Survey of Graph Labeling" [9]. It has considerably greater detail, and in a more formal form, than is presented here.

\section{Graceful labelings}

The universal interest in graph labelings was motivated by Alexander Rosa's $\beta$-valuations [16], which have these ingredients, given a graph $G$ having $m$ edges, with vertex $v$ having label $\ell(v)$ :

Set: $S=\{0,1, \ldots, m\}$

Rule: $f(e)=|\ell(v)-\ell(w)|$

Condition: All edge labels different. 


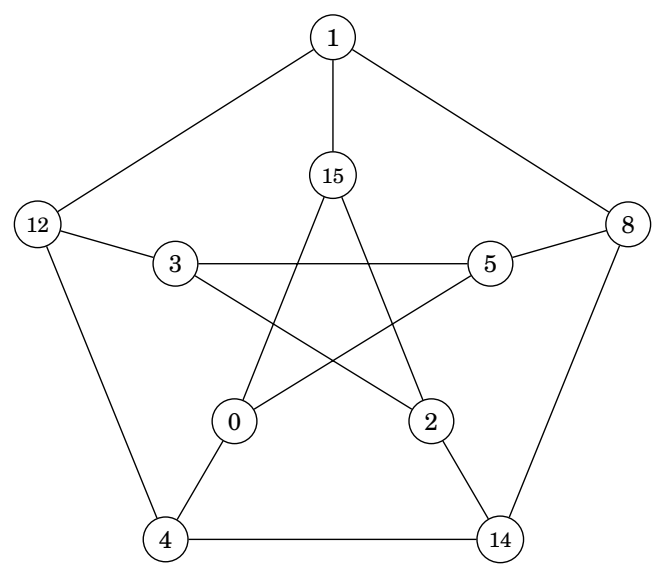

Figure 1: A graceful labeling.

An example of a graceful labeling is shown in Figure 1.

We note that there is a close connection with a conjecture on decompositions of complete graphs into trees, as posed by Gerhard Ringel [15].

\section{Harmonious labelings}

These labelings were introduced and investigated by Ron Graham and Neil Sloane [10]. With the exception of trees, for which the definition is modified slightly, $G$ is a graph with $m$ edges, and here are the ingredients:

For graphs with $m$ edges and edge $e=v w$ :

Set: $S=\{1,2, \ldots, m\}$

Rule: $f(e)=\ell(v)+\ell(w)(\bmod m)$

Condition: All edge labels different

An example of a harmonious labeling is shown in Figure 2.

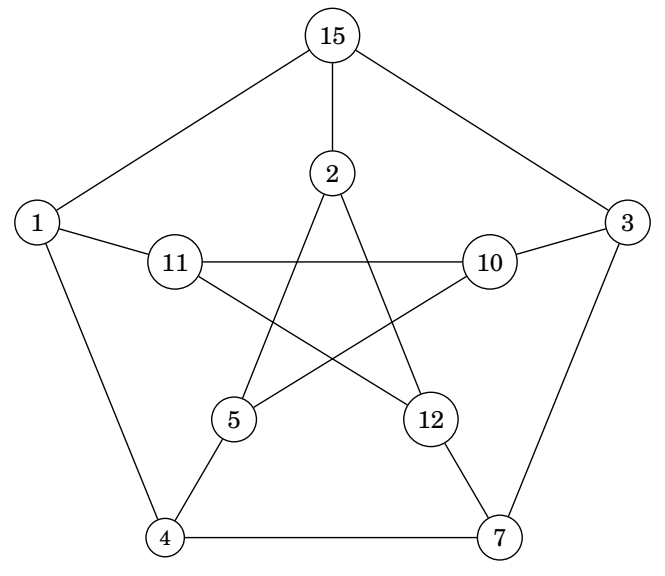

Figure 2: A harmonious labeling.

There is in fact a close connection between harmonious graphs and error-correcting codes (see [10]).

The operations in these labelings are subtraction and addition. In what follows, we consider two other variations; in the first, the operation is multiplication, whereas in the second, the operation is addition but the labels on the vertices must meet a prescribed condition (and the edges are not labeled).

\section{Productive labelings}

In the spirit of Frank Harary, we are re-naming strongly multiplicative graphs as productive graphs (thus fitting in with the names graceful and harmonious). 
As strongly multiplicative graphs, they originated in the paper by Beineke and Hegde [4] with labelings having these ingredients, given a graph of order $n$ :

For graphs of order $n$ and edge $e=v w$ :

Set: $S=\{1,2, \ldots, n\}$

Rule: $f(e)=\ell(v) \cdot \ell(w)$

Condition: All edge labels different

Figure 3 shows that the Petersen graph has a productive labeling.

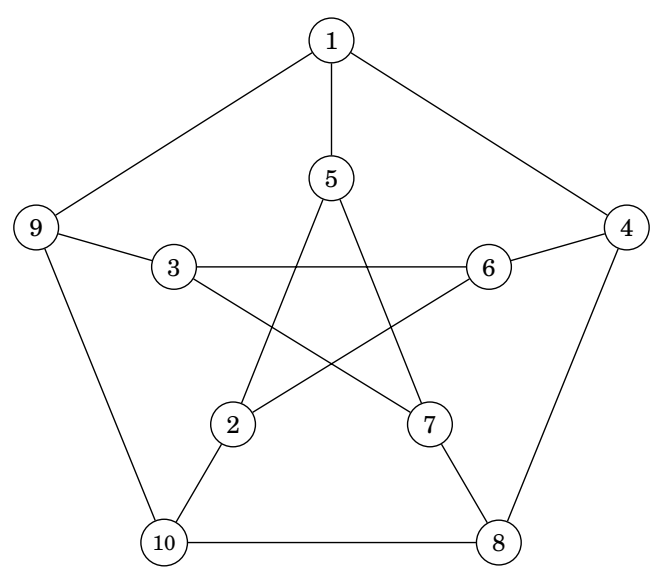

Figure 3: A productive labeling.

The immediate predecessor of productive (strongly multiplicative) labelings was the concept of multiplicative labelings, introduced by B. D. Acharya and S. M. Hegde [1]. These are defined as follows:

For graphs of order $n$ and edge $e=v w$ :

Set: $S=\mathbb{N}$

Rule: $f(e)=\ell(v) \cdot \ell(w)$

Condition: All edge labels different

This type of labeling differs from graceful and harmonious labelings in the unboundedness of the allowable vertex labels being any positive integers. In fact, some restrictions are desirable, as is indicated by the following theorem.

Theorem 2.1. Every graph has a multiplicative labeling.

Proof. Given a graph $G$, label its vertices with different primes. The result is clearly a multiplicative labeling.

This result does however suggest quite an interesting question, but one which we will not explore here: Given a graph $G$, what is the minimum $r$ over all multiplicative labelings of $G$ of the largest label; that is, there is a multiplicative labeling with $S=\{1,2, \ldots, r\}$, but not one with $S=\{1,2, \ldots, r-1\}$.

Returning to productive graphs, we observe that the complete graph $K_{6}$ is not productive since if its vertices are labeled from 1 to 6 , then the edge joining the vertices 1 and 6 clearly gets the same value as that joining 2 and 3 .

The following theorem gives two results on subgraphs and productive graphs.

Theorem 2.2. (a) Every spanning subgraph of a productive graph is productive.

(b) Every graph is an induced subgraph of a productive graph.

Proof. (a) This is a direct consequence of the definition of a productive labeling.

(b) As stated in Theorem 2.1, every graph has a multiplicative labeling. Let $G$ be a graph of order $n$ and let $k$ be the largest label in some multiplicative labeling $\ell$ of $G$. Now take $H$ be the graph obtained from $G$ by adding $k-n$ isolated vertices and labeling them with those positive integers less than $k$ not on vertices of $G$. Then $H$ is clearly productive and has $G$ as an induced subgraph.

Observe that in Part (a) of the theorem the word 'spanning' is necessary, as is shown by the graph in Figure 4 ( $K_{7}$ with two edges at one vertex removed) with a productive labeling. If the vertex labeled 6 is deleted, then what remains is $K_{6}$, 
which, as noted above, is not productive.

\section{Families of productive graphs}

We now consider whether the graphs in some of the well-known and much-studied families of graphs are productive. We begin by showing that not many complete graphs or regular complete bipartite graphs are.

Theorem 2.3. (a) The complete graph $K_{n}$ is productive if and only if $n \leq 5$.

(b) The regular complete bipartite graph $K_{r, r}$ is productive if and only if $r \leq 4$.

Proof. (a) As observed above, $K_{6}$ cannot be productively labeled, while on the other hand, all of the ten products of two integers from 1 to 5 are different. This verifies Part (a).

(b) A productive labeling of $K_{4,4}$ is shown in Figure 4 and deletion of the vertices labeled 7 and 8 results in one for $K_{3,3}$, so all that remains is to show that larger graphs in this family are not productive. Suppose that $K_{r, r}$ is productive, $r \geq 5$. Let $A$ and $B$ be the labels in each of the two disjoint partite sets of five elements each. Let $1 \leq i<j \leq 2 r$. We make two elementary observations: (1) If $i$ and $j$ are both in one of the sets, then $2 i$ and $2 j$ cannot both be in the other set. (2) If $i$ and $2 i$ are both in one of the sets, then $j$ and $2 j$ cannot both be in the other set. It follows that there can be at most two pairs of the form $\{k, 2 k\}$ split between the two sets. Furthermore, all of the other such pairs, so at least $r-2$ of them, must lie in the same set, say $A$. Now let $C=\{1,2, \ldots, r\} \cup\{2,4, \ldots, 2 r\}$. Since $r \geq 5,|C| \geq r+3$. It follows that $B$ then has at most two of the numbers in $C$, and so $|B| \geq r+1$, which is a contradiction. Hence, $K_{r, r}$ cannot be productive.

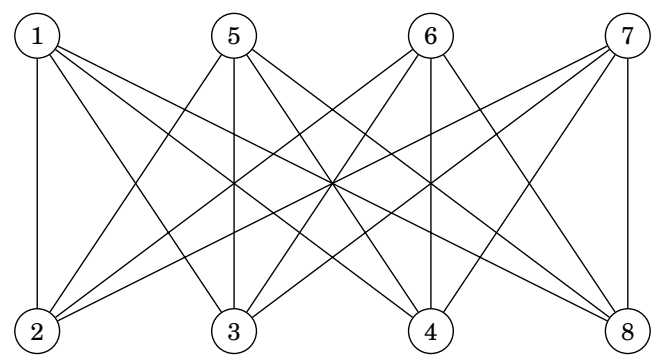

Figure 4: A productive labeling of $K_{4,4}$.

This leaves open the interesting question of which complete bipartite graphs $K_{r, s}$ with $r<s$ are productive; in particular, when $r=3$ and $r=4$.

We turn now to the family of trees. Figure 5 shows one with a productive labeling.

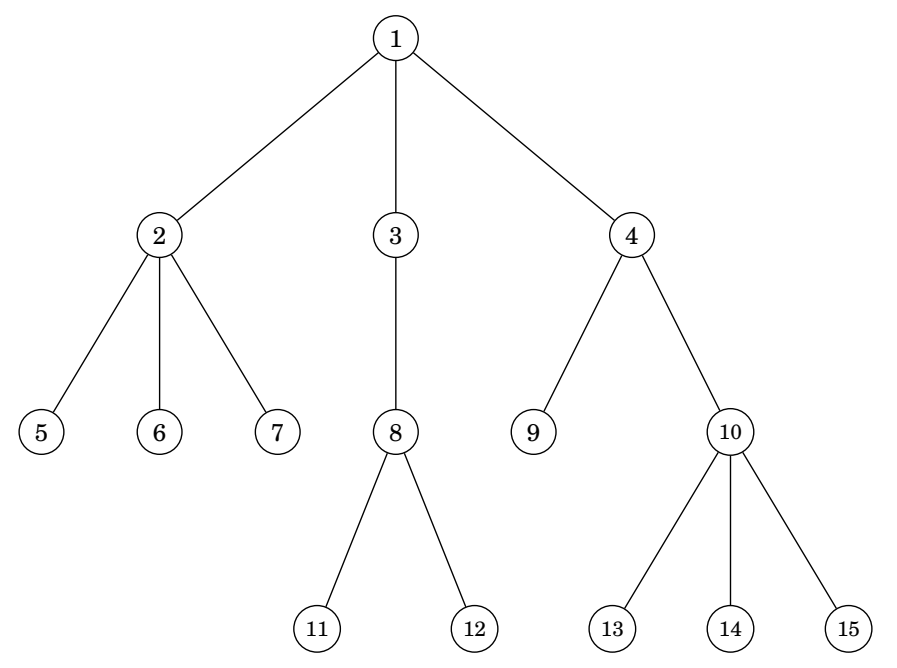

Figure 5: A productive labeling of a tree.

Note that the labeling is that of the vertices encountered in a breadth-first search. It is a straightforward matter to show that this always works, so we have the following result for special productive labelings of trees.

Theorem 2.4. Every tree has a productive labeling in which a given root is labeled 1. 
Turning to cycles, we have the following result; our proof is not quite as simple as one might expect.

Theorem 2.5. Every cycle is productive.

Proof. Consider the cycle $C_{n}$ for $n \geq 3$. It is easy to show that it is productive if $n \leq 6$, so we assume that $n \geq 7$ and let $C=v_{1} v_{2} \ldots v_{n} v_{1}$. Now if $n$ is not the product of two consecutive integers, then the labeling $\ell\left(v_{i}\right)=i$ gives the products on the edges as $1 \cdot 2,2 \cdot 3, \ldots,(n-1) n, n \cdot 1$, and these are clearly all different, so the labeling is productive. Now assume that $n=k(k+1)$, and consider the labeling as previously except that the labels on $v_{k-1}$ and $v_{k}$ are interchanged; that is, $\ell\left(v_{k-1}\right)=k, \ell\left(v_{k}\right)=k-1$, and $\ell\left(v_{i}\right)=i$ otherwise. It is now straightforward to verify that this labeling is also productive.

The same result can be extended to wheels (those graphs $K_{1} * C_{r}$ consisting of a cycle augmented by one vertex adjacent to all of the others); for a proof, the reader is referred to [4].

Theorem 2.6. Every wheel is productive.

The next family that we consider are the grid graphs $P_{r} \times P_{s}$, the products of the path $P_{r}$ of length $r-1$ and the path $P_{s}$ of length $s-1$. Note that its order is $r s$. Using a traditional diagonal pattern of the numbers from 1 to $r s$ it is straightforward to verify that $P_{r} \times P_{s}$ is productive, so we have our next result.

Theorem 2.7. Every grid graph is productive.

The productiveness of powers of paths were investigated by Beineke and Seoud [5]. The $k$ th power $P_{n}^{k}$ of the path $P_{n}$ is obtained from $P_{n}$ by joining all pairs of vertices at distance $k$ or less. The following theorem is a summary of their results for $n \leq 5$.

Theorem 2.8. (a) $P_{n}^{3}$ is productive for all $n$.

(b) $P_{n}^{4}$ is productive except for $n=6$.

(c) $P_{n}^{5}$ is productive if $n \leq 5,9 \leq n \leq 13$, or $n \geq 17$, and is not productive if $n=6,7$, or 8 .

As would be expected from the theorem, whether $P_{n}^{5}$ has a productive labeling when $n=14,15$, or 16 remains unknown. Here are two natural questions based on the limited known results:

1. For each value of $k \geq 4$, does there exist an integer $n_{k}$ for which $P_{n}^{k}$ is productive for all $n \geq n_{k}$ ?

2. For each value of $k \geq 4$, does there exist an integer $n_{k}$ for which $P_{n}^{k}$ is not productive if and only if $6 \leq n<n_{k}$ ?

\section{Maximum numbers of edges}

As we observed at the beginning, when the vertices of $K_{6}$ are labeled 1 to 6 , there are two pairs of edges with the same product, so removing one edge from each pair leaves a productive graph. Because the pairs of products are $1 \cdot 6=2 \cdot 3$ and $2 \cdot 6=3 \cdot 4$, the two chosen edges can be adjacent or they can be independent. Consequently, the removal of any two edges from $K_{6}$ leave a productive graph. Furthermore, since 7 is prime, the same can be said for $K_{7}$. In the context of investigating necessary and sufficient conditions for productive graphs, Seoud and Mahran [17] looked at $K_{8}$ and found that four edges need to be removed in order to have a productive graph. An alternative way of considering this problem is in terms of the greatest number of edges that one can have with a given number of vertices: Let $\lambda(n)$ denote the maximum number of edges in a productive graph of order $n$. Then we have the following table:

\begin{tabular}{c|ccccc}
$n$ & 4 & 5 & 6 & 7 & 8 \\
\hline$\lambda(n)$ & 6 & 10 & 13 & 19 & 24
\end{tabular}

The following result gives upper bounds on the number of edges that one can have.

Theorem 2.9. For all $n$,

$$
\lambda(n) \leq\left\{\begin{array}{lll}
3 n^{2} / 8 & \text { if } n \equiv 0 & (\bmod 4) \\
\left(3 n^{2}+2 n-5\right) / 8 & \text { if } n \equiv 1 & (\bmod 4) \\
\left(3 n^{2}-4\right) / 8 & \text { if } n \equiv 2 & (\bmod 4) \\
\left(3 n^{2}+2 n-9\right) / 8 & \text { if } n \equiv 3 & (\bmod 4)
\end{array}\right.
$$

This is unified in this corollary. 
Corollary 2.1. For $n \geq 12, \lambda(n) \leq 3 n^{2} / 8$.

An alternate view of this function is that $\lambda(n)$ equals the number of different products of two different numbers between 1 and $n$ :

$$
\lambda(n)=|\{r s: 1 \leq r<s \leq n\}| .
$$

Erdős [8] determined its asymptotic behavior:

Theorem 2.10. Let $c=1-\frac{1+\ln \ln 2}{\ln 2}$. Then

$$
\lambda(n) \sim \frac{n^{2}}{(\ln n)^{c+o(1)}} .
$$

An explicit, but quite complicated, formula was found by Kafshar Zaferani [14].

\section{Theorem 2.11.}

$$
\lambda(n)=\frac{n(n-1)}{2}+\sum_{m=2}^{n} \sum_{k=1}^{m-1}\left\lfloor-\frac{\theta(m, k)}{\lfloor\sqrt{m k-1}\rfloor-k+1}\right\rfloor,
$$

where

$$
\theta(m, k)=\sum_{i=k+1}^{\lfloor\sqrt{m k-1}\rfloor}\left\lfloor\frac{\left\lfloor\frac{m k}{i}\right\rfloor}{\frac{m k}{i}}\right\rfloor .
$$

There are of course many other specifications that one can make on multiplicative labelings of graphs, and one of the more interesting are those requiring a geometric sequence for the products, which was investigated by Hegde and Shankaran [11].

\section{Other operations}

A variation on our productive graphs was introduced by Adiga and Somashekara [2] as a family called strongly-* graphs. For graphs of order $n$ and edge $e=v w$ :

Set: $S=\{1,2, \ldots, n\}$

Rule: $f^{*}(e)=\ell(v) \cdot \ell(w)+\ell(v)+\ell(w)$

Condition: All edge labels different

Because of the similarity in its definition to productive graphs, many of the same results hold also for this labeling.

Another interesting variation uses quotients instead of products; strongly quotient graphs were introduced by Adiga, Kafshgar Zaferani, and Smitha [3], and are defined as follows:

For graphs of order $n$ and edge $e=v w$ :

Set: $S=\{1,2, \ldots, n\}$

Rule: $f(e)=\ell(v) / \ell(w)$ with $\ell(v)<\ell(w)$

Condition: All edge labels different

The authors show that the Petersen graph, which we saw was productive, is also strongly quotient, as is shown in Figure 6 .

We note however that this labeling is not productive. Nonetheless, it would appear more likely that a graph is productive than strongly quotient. For, if one edge has labels $a$ and $b$ with $a<b$ and another has labels $c$ and $d$ with $c<d$ it appears more likely that $\frac{a}{b}=\frac{c}{d}$ than that $a b=c d$. This seems to be borne out for some graphs at any rate. For instance, it is known [3], [4] that although $K_{4}$ and $K_{5}$ are productive, they are not strongly quotient, and this is also the case for $K_{3,3}$. In comparing and contrasting the two types of labeling, Adiga, Kafshgar Zaferani, and Smitha [3] prove the following theorems (compare these results with Theorems 2.5, 2.6, and 2.7).

Theorem 2.12. Every cycle is strongly quotient.

Theorem 2.13. A wheel is strongly quotient if and only if it has at least five vertices.

Theorem 2.14. Every grid graph is strongly quotient. 


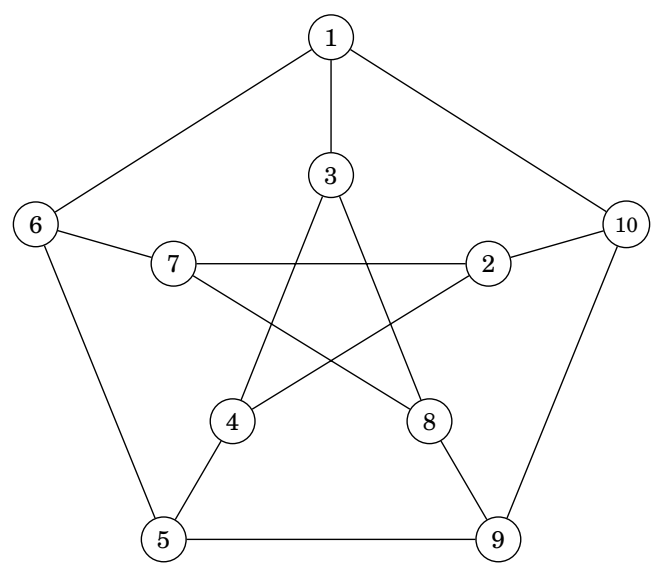

Figure 6: A strongly quotient labeling.

It is interesting that it is an open question whether or not all trees are strongly quotient:

Conjecture. All trees are strongly quotient graphs.

A formula for the greatest number of edges $\mu(n)$ that a strongly quotient graph of order $n$ can have was given in [3]. Interestingly, it involves the Euler $\varphi$-function from number theory.

Theorem 2.15. The maximum number $\mu(n)$ of edges in a strongly quotient graph of order $n \geq 2$ is

$$
\mu(n)=\sum_{k=2}^{n} \varphi(k) .
$$

The following bounds are also known:

Theorem 2.16. For $n \geq 2$,

$$
\sum_{k=2}^{n} \sqrt{k}<\mu(n) \leq \frac{n(n+1)}{2}-\sum_{k=2}^{n-1}\left\lfloor\frac{n}{k}\right\rfloor-2 .
$$

We conclude this section with an open question: Are the $d$-dimensional cubes $Q_{d}$ productive and are they strongly quotient? In Figure 7 we show that the 3-cube has both properties, but with different labelings (neither of these has both properties).
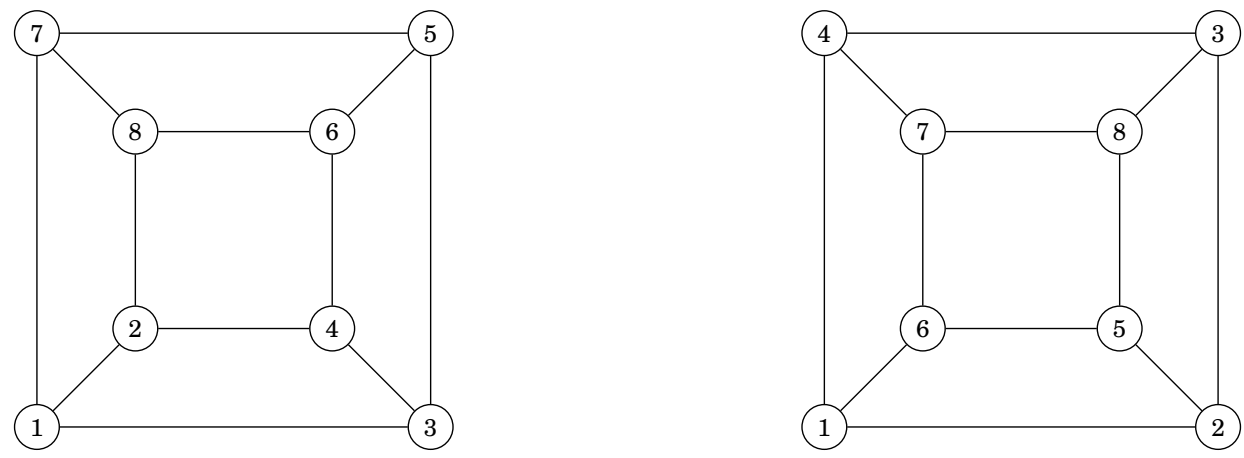

Figure 7: Strongly multiplicative and strongly quotient cubes.

\section{Sum graphs}

Among the many graph-theoretic creations of Frank Harary was that of "sum graphs" within the area of graph labelings. The key concept is that the labels on the vertices are closed under the operation of addition of the labels on adjacent vertices. Originally, Harary [12] restricted the labels to the positive integers, but later extended the possible labels to all integers. The former he called "sum graphs" and the latter "integral sum graphs". Here, we will take the liberty of extending the name "sum graphs" to be the general term and use "positive-valued sum graphs" when that is to be emphasized.

Formally, we have this definition: Given a graph $G$ of order $n$ and a set $S$ of $n$ or more integers, a sum labeling of $G$ is an assignment $\ell(v)$ of different numbers from $S$ to the vertices of $G$ so that if $v w$ is an edge of $G$, then there is a vertex with 
label $\ell(v)+\ell(w)$ in $S$. If all of the labels are positive, the labeling is called positive-valued. We illustrate these definitions in Figure 8. The first graph shows that the path $P_{4}$ has a sum labeling using both positive and negative integers, while the second shows a positive-valued labeling of the union of $P_{3}$ and an isolated vertex. (It is not difficult to see that (and we will show this shortly) that $P_{3}$ itself does not have a positive-valued labeling.)

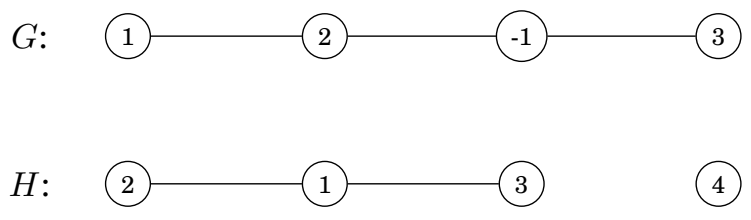

Figure 8: Examples of sum graphs.

In the next two subsections, we look at two of the most interesting aspects of sum graphs that Harary introduced:

- Given a graph $G$, is it a sum graph (for $\mathbb{N}$ or $\mathbb{Z}$ )?

- Given a set $S$ of integers, what is its sum graph?

\section{Features of labelings}

When Harary first introduced positive-valued sum labelings, he observed that no non-trivial connected graph has such a labeling. For, suppose that $G$ is connected and has a labeling with positive integers. Let $v$ be the vertex with the largest label and let $w$ be a vertex adjacent to $v$. Then there cannot be a vertex with label $\ell(v)+\ell(w)$, so $G$ cannot be a positivevalued sum graph. In fact, it follows from this argument that every positive-valued sum graph $G$ must have isolated vertices, and a natural question is how many vertices must such a graph have? For paths, the answer is 1, and Harary used the Fibonacci sequence to illustrate this (see Figure 9).

Harary conjectured that only one vertex was needed for any tree, and this was proved by Ellingham [7].

Theorem 3.1. Every graph consisting of a tree together with one isolated vertex is a positive-valued sum graph.

At the other extreme, Bergstrand et al. [6] showed that for the complete graph $K_{n}$ the minimum number of isolated vertices that are needed to get a positive-valued sum graph is $2 n-3$.

Returning briefly to integer labelings, Harary [13] showed that a Fibonacci-like sequence can be used to show that every path is a sum graph. Consider the sequence $h_{n}$ with $h_{1}=1, h_{2}=2$, and for $n \geq 3, h_{n}=h_{n-2}-h_{n-1}$ (thus the next term is a difference of the two preceding terms rather than a sum). When truncated after $n$ terms, this gives a neat sum labeling of $P_{n}$ since the sum of two adjacent labels is the label of their immediate predecessor. Figure 10 shows this for $n=7$.

We note that given a set of integers $S$, the sum graph of the set of negatives of the elements of $S$ is isomorphic to that of $S$ itself. Most of our discussion will involve labelings with consecutive integers and so we will use the name "interval labelings".

\section{Sum graphs of intervals}

Heretofore the starting point was a graph and the problem was to assign numbers to its vertices so that there was closure under addition. Harary [12] also considered working in the opposite direction: start with a set of numbers and construct a graph that satisfies closure. Formally, if $S$ is a set of $n$ integers, then the sum graph of $S$, denoted $G(S)$, is that graph of order $n$ whose vertices are labeled with the elements in $S$ and two vertices are joined by an edge if their sum is in $S$ (that is, is the label of one of the vertices). In his inaugural paper, Harary chose to feature the graph $G_{n}$ of order $n$ whose vertices are labeled $1,2, \ldots, n$, with the vertices labeled $i$ and $j$ joined by an edge if and only if $i+j \leq n$. We let $G_{n}$ represent this graph and, as others have done, call it the Harary graph of order $n$. From here on, we identify a vertex in a labeled graph with its label. The graphs $G_{6}$ and $G_{7}$ are shown in Figure 11.

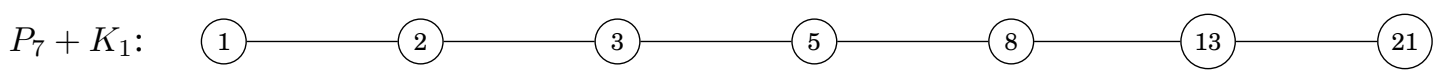

Figure 9: A positive-valued sum graph. 


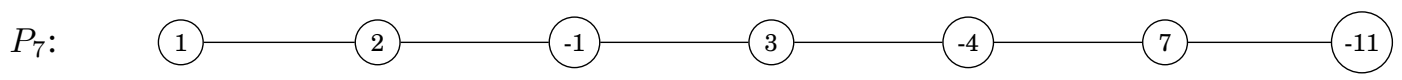

Figure 10: A connected sum graph.
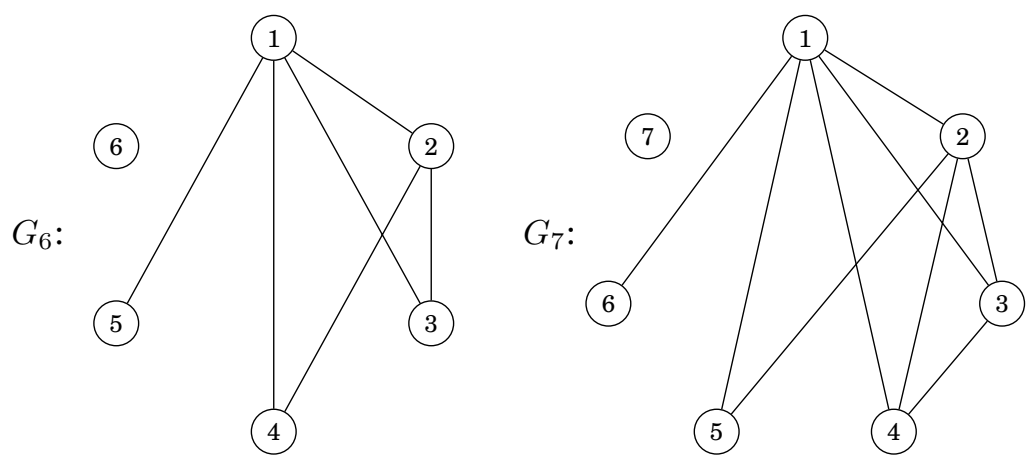

Figure 11: The Harary graphs $G_{6}$ and $G_{7}$.

We observe in this example that the structure of a Harary graph is a split graph, that is, the vertices can be "split" into two sets, one of which induces a complete subgraph and the other induces an independent set of vertices. Further, we observe that the vertex labeled 1 has degree $n-2$, and in going from the vertex labeled $i$ to that labeled $i+1$, the degree goes down by 1 , with one exception, when it remains the same. That exception is for $i=k$ when $n=2 k$ as well as for $i=k+1$ when $n=2 k+1$. Figure 11 illustrates this for the cases $n=6$ and 7 . By summing these degrees, we can deduce the number of edges in $G_{n}$. These facts are summarized in the following result.

Theorem 3.2. (a) For $n \geq 2$, the Harary graph $G_{n}$ is a split graph with the vertices labeled $1,2, \ldots,\left\lceil\frac{n}{2}\right\rceil$ being mutually adjacent and those labeled $\left\lceil\frac{n}{2}\right\rceil+1,\left\lceil\frac{n}{2}\right\rceil+2, \ldots, n$ being mutually independent.

(b) The degree of the vertex with label i in $G_{n}$ is

$$
\operatorname{deg} i= \begin{cases}n-i-1 & \text { if } 1 \leq i \leq\left\lfloor\frac{n}{2}\right\rfloor, \\ n-i & \text { if }\left\lfloor\frac{n}{2}\right\rfloor+1 \leq i \leq n .\end{cases}
$$

(c) $G_{n}$ has $\left\lfloor\frac{(n-1)^{2}}{4}\right\rfloor$ edges.

Another view of the Harary graph $G_{n}$ is to consider, as is often done, a set of consecutive integers in (closed) interval terms; that is, for $r<s$, let $[r, s]$ denote the set $\{r, r+1, \ldots, s\}$. Then $G_{n}$ is the sum graph $G([1, n])$. For $r>0$, we denote the sum graph of the interval $[r+1, r+n]$ as $G_{r+1, r+n}$. The following theorem of Vilfred, Beineke, and Suryakala [18] extends Theorem 3.2 to these graphs. We note that our notation for graph operations differs from that of some authors. For the disjoint union of two graphs $G$ and $H$, we write $G+H$ (this is consistent in having $G+G=2 G$ ), and for the join (in which every vertex of $G$ is joined to every vertex of $H$ ), we write $G * H$.

Theorem 3.3. Let $r \geq 0$ and $n \geq 1$.

(a) For $r \leq n-3, G_{r+1, r+n} \cong G_{n-r}+r K_{1}$, while for $r \geq n-2$, it is totally disconnected.

(b) For $r \leq n-3$, the interval graph $G_{r+1, r+n}$ is a split graph with the vertices labeled $r+1, r+2, \ldots,\left\lceil\frac{n}{2}\right\rceil$ being mutually adjacent and those labeled $\left\lceil\frac{n}{2}\right\rceil+1,\left\lceil\frac{n}{2}\right\rceil+2, \ldots, n$ being mutually independent.

(c) The degree of the vertex with label $i$ in $G_{r+1, r+n}$ is

$$
\operatorname{deg}(i+r)= \begin{cases}n-r-i-1 & \text { if } 1 \leq i \leq\left\lfloor\frac{n-r}{2}\right\rfloor, \\ n-r-i & \text { if }\left\lfloor\frac{n-r}{2}\right\rfloor+1 \leq i \leq n-r . \\ 0 & \text { otherwise. }\end{cases}
$$

(d) $G_{r+1, r+n}$ has $\left\lfloor\frac{(n-r-1)^{2}}{4}\right\rfloor$ edges. 
The extension of this concept to all intervals of integers was introduced by Vilfred and Mary Florida [19], with the most interesting being those with both positive and negative values. Note that if every label in a sum graph $G(S)$ is replaced by its negative, the resulting sum graph is isomorphic to the original sum graph. Hence, we assume for convenience (unless it is specified otherwise), that sum graphs have at least as many positive as negative labels. Thus, for an interval with both positive and negative values, we adopt the notation $[-r, s]$ with $1 \leq r \leq s$. Clearly, the sum graph of such an interval contains 0 and hence is connected. Figure 12 shows the sum graphs of these intervals of length 6 .

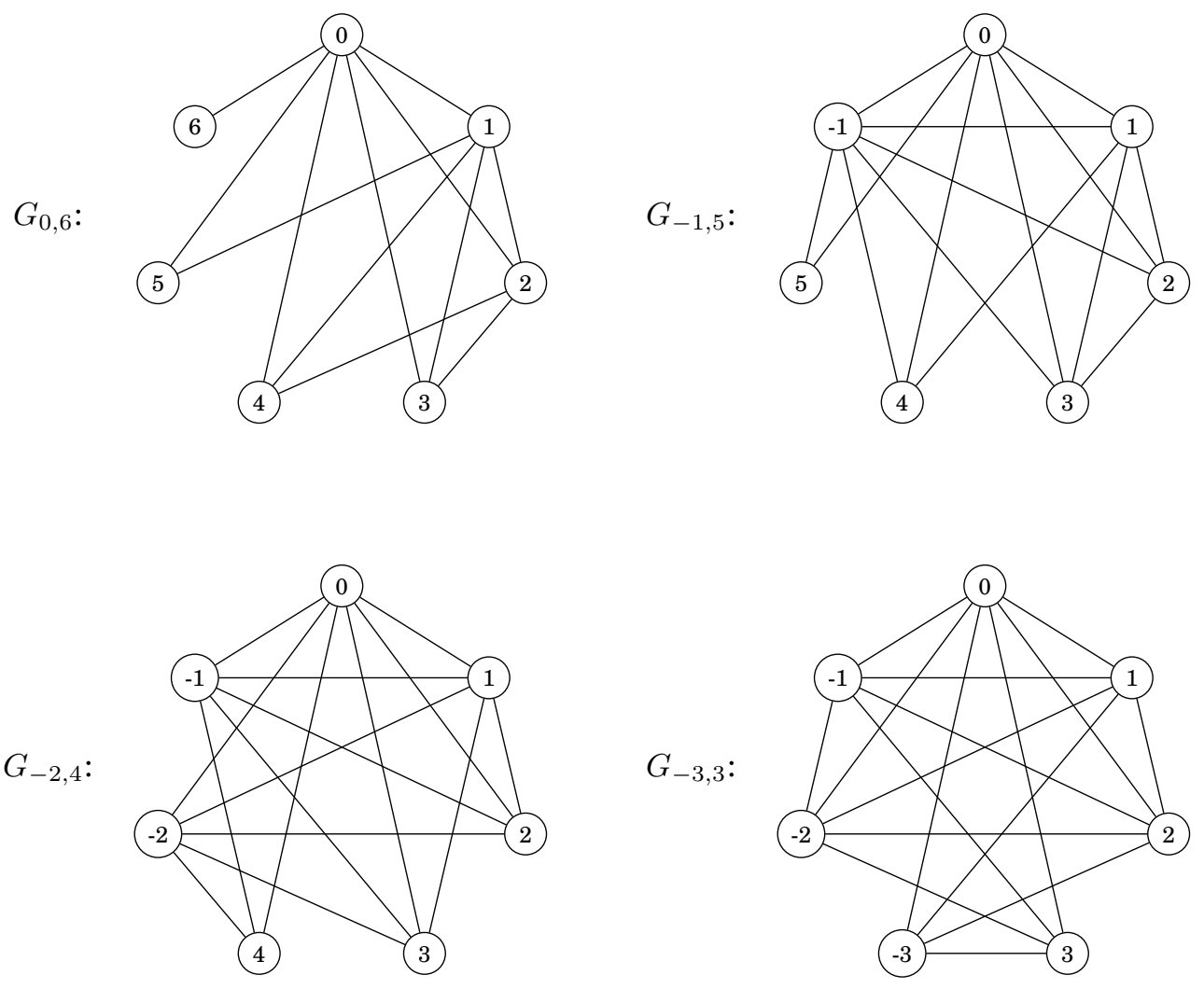

Figure 12: Sum graphs of intervals.

These sum graphs of intervals differ from the earlier ones in that not all of them are split graphs. In fact, each of the vertices labeled with a negative number will be adjacent to each of those with a positive label. In other words, the set of positive vertices and and the set of negative vertices yield a complete bipartite subgraph (in general not induced). As a generalization of a split graph, we define a 3-way split graph to be a graph whose vertices can be partitioned three subsets, one of which induces a complete graph and each of the other two forms an independent set. The following theorem summarizes the main properties of the family of sum graphs of intervals with both positive and negative labels.

Theorem 3.4. Let $1 \leq r \leq s$ and $n=r+s+1$.

(a) The sum graph $G_{-r, s} \cong K_{1} * G_{r} * G_{s}$.

(b) $G_{-r, s}$ is a 3-way split graph with the vertices labeled $-\left\lceil\frac{r}{2}\right\rceil,-\left\lceil\frac{r}{2}\right\rceil+1, \ldots,-1,0,1, \ldots,\left\lceil\frac{s}{2}\right\rceil$ being mutually adjacent and those labeled in each of the groups $-\left\lceil\frac{r}{2}\right\rceil+1,-\left\lceil\frac{r}{2}\right\rceil+2, \ldots,-r$ and $\left\lceil\frac{s}{2}\right\rceil+1,\left\lceil\frac{s}{2}\right\rceil+2, \ldots$, s being mutually independent.

(c) In $G_{-r, s}$ the degree of the vertex with label $i$ is

$$
\operatorname{deg} i= \begin{cases}n+i & \text { if }-r \leq i \leq-\left\lfloor\frac{r}{2}\right\rfloor-1, \\ n+i-1 & \text { if }-\left\lfloor\frac{r}{2}\right\rfloor \leq i \leq-1, \\ n-1 & \text { if } i=0 \\ n-i-1 & \text { if } 1 \leq i \leq\left\lfloor\frac{s}{2}\right\rfloor \\ n-i & \text { if }\left\lfloor\frac{s}{2}\right\rfloor+1 \leq i \leq s\end{cases}
$$

(d) $G_{-r, s}$ has $r s+r+s+\left\lfloor\frac{(r+1)^{2}}{4}\right\rfloor+\left\lfloor\frac{(s+1)^{2}}{4}\right\rfloor$ edges.

With that, with a final recognition of Frank Harary's tremendous contributions to graph theory, we conclude this survey of two beautiful types of graph labelings. 


\section{References}

[1] B. D. Acharya, S. M. Hegde, On certain vertex valuations of a graph, Indian J. Pure Appl. Math. 22 (1991) 553-560.

[2] C. Adiga, M. Smitha, An upper bound for maximum number of edges in a strongly multiplicative graph, Discuss. Math. Graph Theory 26 (2006).

[3] C. Adiga, R. K. Zaferani, M. Smitha, Strongly quotient graphs, South East Asian J. Math. 5 119-127.

[4] L. W. Beineke, S. M. Hegde, Strongly Multiplicative Graphs, Discuss. Math. Graph Theory 21 (2001) 63-76.

[5] L. W. Beineke, M. A. Seoud, On the strong multiplicativity of powers of paths, Congr. Numer. 131 (1998) 211-217.

[6] D. Bergstrand, K. Hodges, G. Jennings, L. Kuklinski, J. Wiener, F. Harary, The sum number of a complete graph, Bull. Malaysian Math. Soc. 12 (1989) 25-28.

[7] M. N. Ellingham, Sum graphs from trees, Ars Combin. 35 (1993) 335-349.

[8] P. Erdős, An asymptotic inequality in the theory of numbers, Vestnik Leningrad Univ. 15 (1960) 41-49.

[9] J. A. Gallian, A dynamic survey of graph labeling, Electron. J. Combin. (2019) Art\# DS6.

[10] R. L. Graham, N. J. A. Sloane, On additive bases and harmonious graphs, SIAM J. Algebr. Discrete Methods 1 (1980) $382-404$.

[11] S. M. Hegde, P. Shankaran, Geometric labeled graphs, AKCE J. Graphs Combin. 5 (2008) 83-97.

[12] F. Harary, Sum graphs and difference graphs, Congr. Numer. 72 (1990) 101-108.

[13] F. Harary, Sum graphs over all integers, Discrete Math. 124 (1994) 99-105.

[14] R. Kafshgar Zaferani, The maximum number of edges in a strongly multiplicative graph, Bull. Iranian Math. Soc. 31 (2005) 53-56.

[15] G. Ringel, Problem 25, In: Theory of Graphs and its Applications (Proc. Int. Symp. Smolenice 1963), Czech Acad. Sci., Prague, 1964, p.162.

[16] A. Rosa, On certain valuations of the vertices of a graph, In: Theory of Graphs (Int. Symp. Rome 1966), Gordon and Breach, N. Y. and Dunod Paris, 1967, pp. 349-355.

[17] M. A. Seoud, A. E. A. Mahran, On strongly multiplicative graphs, Ars Combin. 118 (2015) 155-165.

[18] K. Vilfred, L. W. Beineke, A. Suryakala, More properties of sum graphs, Graph Theory Notes N. Y. 66 (2014) 10-15.

[19] K. Vilfred, L. M. Florida, Integral sum graphs and maximal integral sum graphs, Graph Theory Notes N. Y. 63 (2012) $28-36$. 